\title{
ON THE RELATIONSHIP BETWEEN A SUMMABILITY MATRIX AND ITS TRANSPOSE
}

\author{
J. SWETITS
}

(Received 21 June 1977; revised 5 February 1979)

Communicated by A. P. Robertson

\begin{abstract}
Let $E, F$ be sequence spaces and $A$ an infinite matrix that maps $E$ to $F$. Sufficient conditions are given so that the transposed matrix maps $F^{\beta}$ to $E^{\beta}$.

1980 Mathematics subject classification (Amer. Math. Soc.): primary 40 C 05, 40 D 25; secondary $40 \mathrm{H} 05$.
\end{abstract}

\section{Introduction}

Let $A$ be an infinite matrix of complex numbers and $A^{\prime}$ its transpose. Vermes (1957) considered the relationships between $A$, as a regular sequence to sequence summability method, and $A^{\prime}$, as a regular series to series method. Jakimovski and Russell (1972) obtained some additional results on the relationships between $A$ and $A^{\prime}$, when $A$ is a mapping between $B K$ spaces.

In this note we consider $A$ as a mapping between two sequence spaces, $E$ and $F$, and determine when $A^{\prime}$. maps $F^{\beta}$ to $E^{\beta}$. The range of corollaries includes some of the results of Jakimovski and Russell (1972), a result of Skerry (1974), and a result related to one announced by Dawson (1976).

\section{Preliminaries}

A sequence space is a vector subspace of the space $\omega$ of all complex sequences. A sequence space $E$ with a locally convex topology, $\tau$, is a $K$ space if the linear functionals

$$
x \rightarrow x_{j}, \quad j=0,1,2, \ldots,
$$


are continuous. In addition, if $(E, \tau)$ is complete and metrizable, then $E$ is an $F K$ space. A normed $F K$ space is a $B K$ space.

If $E$ is a sequence space, we write

$$
\begin{aligned}
& E^{\beta}=\left\{y \in \omega: \sum_{j=0}^{\infty} x_{j} y_{j} \text { converges for all } x \in E\right\}, \\
& E^{\alpha}=\left\{y \in \omega: \sum_{j=0}^{\infty}\left|x_{j} y_{j}\right|<\infty \text { for all } x \in E\right\}, \\
& E^{\gamma}=\left\{y \in \omega: \sup _{n}\left|\sum_{j=0}^{n} x_{j} y_{j}\right|<\infty \text { for all } x \in E\right\} .
\end{aligned}
$$

Let $\varphi$ be the space of sequences with only finitely many non-zero terms. In this paper, it will be assumed that all sequence spaces contain $\varphi$.

If $F$ is a subspace of $E^{\beta}$, then $E$ and $F$ form a dual pair under the bilinear form

$$
\langle x, y\rangle=\sum_{j=0}^{\infty} x_{j} y_{j} .
$$

The weak topology on $E$ by $F, \sigma(E, F)$, is a $K$ space topology. Topologies for dual pairings of the type described above have been considered by Garling (1967a).

If $x \in \omega$, let $P_{n} x=\left\{x_{0}, x_{1}, \ldots, x_{n}, 0,0, \ldots\right\}$. If $(E, \tau)$ is a $K$ space such that $P_{n} x \rightarrow x$ for each $x \in E$, then $E$ is called an $A K$ space.

If $A=\left(a_{n k}\right)$ is an infinite matrix of complex numbers the sequence $A x=\left\{(A x)_{n}\right\}$ is defined by

$$
(A x)_{n}=\sum_{k=0}^{\infty} a_{n k} x_{k}, \quad n=0,1,2, \ldots
$$

$E_{A}=\{x \in \omega: A x \in E\}$, where $E$ is a sequence space. Also $A^{\prime}$ denotes the transpose of $A$.

The following spaces will be used in the sequel:

$$
\begin{aligned}
m & =\left\{x \in \omega: \sup _{n}\left|x_{n}\right|<\infty\right\} ; \\
c_{0} & =\left\{x \in \omega: \lim _{n \rightarrow \infty} x_{n}=0\right\} ; \\
l^{p}(1 \leqslant p<\infty) & =\left\{x \in \omega: \sum_{n=0}^{\infty}\left|x_{n}\right|^{p}<\infty\right\} ; \\
b s & =\left\{x \in \omega: \sup _{n}\left|\sum_{k=0}^{n} x_{k}\right|<\infty\right\} ; \\
b v & =\left\{x \in \omega: \sum_{n=0}^{\infty}\left|x_{n}-x_{n+1}\right|<\infty\right\} ;
\end{aligned}
$$




$$
\begin{aligned}
b v_{0} & =b v \cap c_{0} \\
c s & =\left\{x \in \omega: \sum_{n=0}^{\infty} x_{n} \text { converges }\right\} .
\end{aligned}
$$

Each of the above is a $B K$ space when topologized in the usual way. In addition, all except $b s, m$ and $b v$ are $A K$ spaces.

It is well known that $\left(l^{p}\right)^{\beta}=l^{q},(1 / p)+(1 / q)=1$ and $p \neq 1 ; l^{\beta}=m ; m^{\beta}=l$; $b v_{0}^{\beta}=b s ; b s^{\beta}=b v_{0} ; b v^{\beta}=c s ; c s^{\beta}=b v ; c s^{\gamma}=b v ; b v^{\gamma}=b s$ and $c_{0}^{\beta}=l$.

\section{Main results}

Let $E$ be a sequence space containing $\varphi$ such that $\left(E^{\beta}, \sigma\left(E^{\beta}, E\right)\right)$ is sequentially complete. Let $B=\left(b_{n k}\right)$ be an infinite matrix such that $\left\{(B x)_{n}\right\}$ is convergent for every $x \in E$. For each $n=0,1,2, \ldots$, let $b^{(n)}=\left\{b_{n k}\right\}_{k=0}^{\infty}$. Then $\left\{b^{(n)}\right\}$ is a Cauchy sequence in $\left(E^{\beta}, \sigma\left(E^{\beta}, E\right)\right)$. Thus, there exists $b=\left\{b_{k}\right\} \in E^{\beta}$ such that

$$
\lim _{n \rightarrow \infty}(B x)_{n}=\sum_{k=0}^{\infty} b_{k} x_{k}
$$

for every $x \in E$. Since $E$ contains $\varphi$ it follows that, for each $k=0,1,2, \ldots$,

$$
\lim _{n \rightarrow \infty}\left(B e^{k}\right)_{n}=b_{k},
$$

where $e^{k}$ denotes the sequence with a one in the $k$ th coordinate and zeroes elsewhere.

These considerations provide the key to the following theorem. The complete proof may be found in Swetits (1978), Theorem 2.1.

THEOREM 3.1. Let $E$ and $F$ be sequence spaces, each containing $\varphi$, such that $\left(E^{\beta}, \sigma\left(E^{\beta}, E\right)\right)$ and $\left(F, \sigma\left(F, F^{\beta}\right)\right)$ are sequentially complete. If $A=\left(a_{n k}\right)$ is an infinite matrix then the following are equivalent:

(i) $F_{A}$ contains $E$;

(ii) $E_{A^{\prime}}^{\beta}$ contains $F^{\beta}$;

(iii) $F_{A}$ contains $\left(E^{\beta}\right)^{\beta}$.

If the hypotheses in Theorem 3.1 are omitted, then the conclusions can fail. Define $A=\left(a_{n k}\right)$ by

$$
a_{n k}=\left\{\begin{aligned}
1, & k=n \\
-1, & k=n+1 \\
0, & \text { otherwise }
\end{aligned}\right.
$$

Then $l_{A}$ contains $b v$. However, $c s$ is not $\sigma(c s, b v)$ sequentially complete and $c s_{A}$, does not contain $m$. Thus, (i) $\rightarrow$ (ii) fails. 
Let $B=A^{\prime}$ where $A$ is the matrix defined above. Then $c s_{B}$ contains $c_{0}, l_{B}$. contains $b v$, but $c s_{B}$ does not contain $m$. Thus, (ii) $\Rightarrow$ (ii) fails.

Examples of spaces, $E$, that satisfy the conditions of Theorem 3.1 are monotone spaces (that is, the coordinatewise product $x y \in E$ if $x \in E$ and $y$ is a sequence of zeroes and ones (Bennett (1974), p. 55)), $F K-A K$ spaces, and Garling's class of $B_{0}$ invariant spaces (Garling (1967b)).

Examples of spaces, $F$, that satisfy the conditions of Theorem 3.1 are perfect spaces $\left(F=\left(F^{\alpha}\right)^{\alpha}\right), b s, b v$ and $b v_{0}$. Each of the spaces mentioned in Section 2 is in one of the above categories.

The first corollary to Theorem 3.1 is well known. For each $p, l \leqslant p \leqslant \infty, l^{p}$ is a perfect space.

CoRollary 3.2. $\left(l^{p}\right)_{A}$ contains $l^{q}$ if and only if $\left(l^{q^{\prime}}\right)_{A^{\prime}}$ contains $l^{p^{\prime}}$, where $(1 / p)+\left(1 / p^{\prime}\right)=1$ and $(1 / q)+\left(1 / q^{\prime}\right)=1$.

A sequence $x$ is said to be entire if $\sum_{n=0}^{\infty}\left|x_{n}\right| p^{n}<\infty$ for all $p>0$. $x$ is analytic if $\sum_{n=0}^{\infty}\left|x_{n}\right| p^{n}<\infty$ for some $p>0$. Let $\mathscr{E}$ be the space of entire sequences and $\mathscr{A}$ the space of analytic sequences. Then $\mathscr{E}^{\beta}=\mathscr{A}$ and $\mathscr{A}^{\beta}=\mathscr{E}$, and both $\mathscr{E}$ and $\mathscr{A}$ are perfect spaces. The following result has been obtained by Skerry (1974), Theorem 4.5.

COROLlaRY 3.3. $\mathscr{E}_{A}$ contains $\mathscr{E}$ if and only if $\mathscr{A}_{A}^{\prime}$ contains $\mathscr{A}$.

Macphail (1951), Theorem 2, established necessary and sufficient conditions for a matrix $A=\left(a_{n k}\right)$ to transform every analytic sequence into $l$. His result, combined with Theorem 3.1, yields

COROLlary 3.4. $\mathscr{E}_{A}$ contains $m$ if and only if, for every $r>0$, there is a constant $M(r)$ such that

$$
\sum_{k=0}^{\infty}\left|a_{n k}\right|<M(r) r^{n}, \quad n=0,1,2, \ldots
$$

The next two corollaries are stated in Jakimovski and Russell (1972), p. 352. They are consequences of Theorem 3.1, (i) $\Rightarrow$ (ii).

COROLlary 3.5. If $c_{A}$ contains $c_{0}$, then $l_{A^{\prime}}$ contains $l$.

COROLLARY 3.6. If $c_{A}$ contains $b v_{0}$, then $b s_{A^{\prime}}$ contains $l$.

The next result enlarges the class of spaces, $F$, for which the equivalence between (i) and (iii) of Theorem 3.1 is valid. 
THEOREM 3.7. Let $E, F$ be sequence spaces, each containing $\varphi$, such that $\left(E^{\beta}, \sigma\left(E^{\beta}, E\right)\right)$ is sequentially complete and $F=\left(F^{\gamma}\right)^{\gamma}$. If $F_{A}$ contains $E$, then $F_{A}$ contains $\left(E^{\beta}\right)^{\beta}$.

Proof. Let $\left\{t_{k}\right\} \in F^{\gamma}$ and $\left\{x_{k}\right\} \in E$. Then

This means

$$
\sup _{j}\left|\sum_{n=0}^{j} t_{n} \sum_{k=0}^{\infty} a_{n k} x_{k}\right|<\infty .
$$

$$
\sup _{j}\left|(B x)_{j}\right|<\infty,
$$

where $B=\left(b_{j k}\right)$ is defined by

$$
b_{j k}=\sum_{n=0}^{j} t_{n} a_{n k}
$$

Thus $m_{B}$ contains $E$. Since $(m, \sigma(m, l))$ is sequentially complete, Theorem 3.1 implies that $m_{B}$ contains $\left(E^{\beta}\right)^{\beta}$. Thus, for any $x \in\left(E^{\beta}\right)^{\beta}$,

$$
\sup _{j}\left|\sum_{n=0}^{j} t_{n} \sum_{k=0}^{\infty} a_{n k} x_{k}\right|<\infty .
$$

It follows that $A x \in\left(F^{\nu}\right)=F$. Hence $F_{A}$ contains $\left(E^{\beta}\right)^{\beta}$.

The following corollary is immediate.

Corollary 3.8. Let $F$ be as in Theorem 3.1 or Theorem 3.7. If $F_{A}$ contains $c_{0}$, then $F_{A}$ contains $m$.

The space of convergent quasiconvex sequences of order $r$, c.q.s. $(r)$ is $\operatorname{drfined}$ as follows: $x \in$ c.q.s. $(r)$ if

where

$$
\sum_{k=0}^{\infty}\left(\begin{array}{c}
k+r-1 \\
k
\end{array}\right)\left|\Delta^{r} x_{k}\right|<\infty
$$

$$
\Delta^{r} x_{k}=\sum_{n=0}^{r}(-1)^{n}\left(\begin{array}{l}
r \\
n
\end{array}\right) x_{k+n}
$$

Jakimovski and Livne (1972), Theorem 4.2, have characterized those matrices, $A$, such that $c_{A}$ contains c.q.s. $(r)$. Using their result, it is an easy matter to verify that ((c.s.q. $\left.(r))^{y}\right)^{\gamma}=$ c.q.s. $(r)$. With $F=$ c.q.s. $(r)$, Corollary 3.8 is closely related to a result recently announced by Dawson (1976).

For any $B K$ space $E$, define

$$
\|y\|_{E^{\nu}}=\sup _{n} \sup _{\|x\|_{E} \leqslant 1}\left|\sum_{k=0}^{n} x_{k} y_{k}\right|<\infty .
$$


If $E, T$ are $B K$ spaces and $A$ is a matrix, let

$$
\|A\|_{(E, F)}=\sup _{p} \sup _{q} \sup _{\|x\|_{E} \leqslant 1} \sup _{\|y\|_{F^{\nu} \leqslant 1}}\left|\sum_{j=0}^{q} y_{j} \sum_{k=0}^{j} a_{j k} x_{k}\right| .
$$

Jakimovski and Livne (1971), Theorem 5.2, have shown that, if $E$ is a $B K-A K$ space and $F=G^{\gamma}$ where $G$ is a $B K$ space, then $F_{A}$ contains $E$ if and only if $\|A\|_{(E, F)}<\infty$. This result, combined with Theorem 3.7 , yields

Corollary 3.9. Let $E$ be a $B K-A K$ space and $F=G^{\gamma}$ where $G$ is a $B K$ space. Then $F_{A}$ contains $\left(E^{\beta}\right)^{\beta}$ if and only if $\|A\|_{(E, F)}<\infty$.

In Corollary $3.9,\left(E^{\beta}\right)^{\beta}$ cannot be replaced by $\left(E^{\gamma}\right)^{\gamma}$. Let $E=c s$ and $F=l$. Then $\left(c s^{\gamma}\right)^{\gamma}=b s$. Let $A$ be the matrix whose first row consists entirely of ones and all of whose other entries are zero. Then $l_{A}=c s$.

A special case of Corollary 3.9 is the well-known equivalence of the following:

(i) $m_{A}$ contains $c_{0}$;

(ii) $m_{A}$ contains $m$;

(iii) $\sup _{n} \sum_{k=0}^{\infty}\left|a_{n k}\right|<\infty$.

A $B K$ space $E$ has the property $F A K$ if $\left\{f\left(P_{n} x\right)\right\}$ converges for every $x \in E$ and every continuous linear functional, $f$, on $E$. $E$ has the property $A B$ if $\left\{\left\|P_{n} x\right\|\right\}$ is bounded for each $x \in E$ (see Zeller (1951); Sargent 1964)). It is known that $F A K$ implies $\boldsymbol{A} \boldsymbol{B}$.

Let $E_{0}$ be the closure in $E$ of $\varphi$. If $E$ has $A B$, then $E_{0}$ is a $B K-A K$ space with the norm of $E$ (Sargent (1964), Theorem 2). Sargent (1964), Theorem 3, has shown that $E$ has $F A K$ if and only if $E_{0}^{\beta}=E^{\beta}$. Combining these results with Corollary 3.9 we have

COROLlary 3.10. Let $E$ be a $B K-F A K$ space, $E_{0}$ the closure in $E$ of $\varphi$, and $F=G^{\gamma}$ where $G$ is a $B K$ space. Then $F_{A}$ contains $E$ if and only if $\|A\|_{\left(E_{0}, F\right)}<\infty$.

Corollary 3.10 cannot be extended to $B K-A B$ spaces. Let $E=b s, E_{0}=c s$, $F=l$, and use the example following Corollary 3.9 .

Finally, it is noted that Theorem 3.1 proved useful in characterizing dense barrelled subspaces of an $F K-A K$ space (Swetits (1978)).

\section{References}

G. Bennett (1974), 'A new class of sequence spaces with applications in summability theory', J. Reine Angew. Math. 266, 49-75. 
D. F. Dawson (1976), 'Matrix maps of null sequences', Notices Amer. Math. Soc. 23, A-134, Abstract No. 731-40-2.

D. J. H. Garling (1967a), 'The $\beta$ and $\gamma$ duality of sequence spaces', Proc. Camb. Phil. Soc. 63, 963-981.

D. J. H. Garling (1967b), 'On topological sequence spaces', Math. Proc. Cambridge Philos. Soc. 63, 997-1019.

A. Jakimovski and A. Livne (1971), 'General Kojima-Topelitz like theorems and consistency theorems', J. Analyse Math. 24, 323-368.

A. Jakimovski and A. Livne (1972), 'On matrix transformations between sequence spaces', $J$. Analyse Math. 25, 345-370.

A. Jakimovski and D. C. Russell (1972), 'Matrix mappings between BK spaces', Bull. London Math. Soc. 4, 345-353.

M. S. Mcphail (1951), 'Some theorems on absolute summability', Canad. J. Math. 3, 386-390.

W. L. C. Sargent (1964), 'On sectionally bounded BK spaces', Math. Zei(. 83, 57-66.

H. B. Skerry (1974), 'On matrix maps of entire sequences', Pacific J. Math. 51, 563-570.

J. Swetits (1978), 'A characterization of a class of barrelled sequence spaces', Glasgow Math. J. 19, 27-31.

P. Vermes (1957), 'The transpose of a summability matrix', Coll. Théorie des Suites (Bruxelles), $60-86$.

K. Zeller (1951), 'Abschnittskonvergenz in FK-Räumen', Math. Zeit. 55, 55-70.

Department of Mathematical and Computing Sciences

Old Dominion University,

Norfolk, Virginia 23508

USA 